\title{
Normal intracranial BS/BSOB ratio values in the first trimester of single gestations with live fetuses in a Korean population
}

\author{
Sung Hee Yang ${ }^{1}$, Hyun Sook An ${ }^{1}$, Jin-Soo Lee ${ }^{2}$, Changsoo Kim ${ }^{3}$
}

${ }^{1}$ Department of Obstetrics and Gynecology, Ilsin Christian Hospital, ${ }^{2}$ Department of Radiology, Haeundae Paik Hospital, Inje University College of Medicine, ${ }^{3}$ Department of Radiological Science, College of Health Sciences, Catholic University of Pusan, Busan, Korea

\begin{abstract}
Aim: The purpose of this study was to determine the normal length of the brainstem (BS) in Korean fetuses and to evaluate the usefulness of the routine measurement of BS size in the first trimester of pregnancy for the early detection of spina bifida. Material and methods: A total of 2,621 normal singleton pregnant Korean women at $10^{+6}$ to $13^{+6}$ weeks of gestation were selected for this retrospective cross-sectional study. Ultrasonography was used to measure the length of the longest vertical depth diameter of the BS and brainstem-occipital bone (BSOB) in order to obtain the BS to BSOB ratio. Results: The best indicators for spina bifida ranged from $1.00 \pm 0.24 \mathrm{~mm}$ to $4.70 \pm 0.46 \mathrm{~mm}$ for the BS and from $2.90 \pm 0.36 \mathrm{~mm}$ to $8.50 \pm 0.92 \mathrm{~mm}$ for the BSOB. For the gestational period, BS $(\mathrm{R}=0.70)$ and $\mathrm{BSOB}(\mathrm{R}=0.81)$ values were considered statistically significant $(\mathrm{p}<.0001)$. The value of the BS to BSOB ratio was $<1.0$ in normal fetuses, and was not correlated with the gestational age. Conclusion: Measurement of BS and BSOB diameter in the first trimester is thought to provide the best reference marker for evaluating the posterior brain for diagnosis of spina bifida.
\end{abstract}

Keywords: gestational age; spina bifida; brainstem; brainstem-occipital bone.

\section{Introduction}

Spina bifida, the most common type of neural tube defect (NTD), occurs in approximately 1 to 2 out of 2,000 newborn babies, and is one of the open fetal malformations [1]. An NTD occurs when the neural tube is not completely closed along the spine because of the derangement of the normal embryogenic cascade at 2 to 4 weeks of gestation [2].

In screening for spina bifida, pregnant patients are subjected to ultrasonography (US) as well as an alphafetoprotein (AFP) test determined in a sample of maternal serum in the second trimester. Screening for NTB is

Received 11.11.2016 Accepted 22.01.2017

Med Ultrason

2017, Vol. 19, No 2, 190-194

Corresponding author: Changsoo Kim

Department of Radiological Science,

College of Health Sciences, Catholic

University of Pusan, 57, Oryundae-ro,

Geumjeong-gu, Busan, Korea 46252

Phone: +82-51-510-0580, Fax: +82-81-510-0589

E-mail: cszzim@cup.ac.kr based on positive readings using the cut-off level of 2.02.5 multiples of the median (MoM). Additionally, both an amniotic fluid acetyl-cholinesterase (AChE) test and target US are conducted for diagnostic evaluation $[3,4]$.

Diagnosis of spina bifida in the first trimester is commonplace and it was recently reported that the false positive rate in the AFP test is much lower when it is conducted in the first trimester comparing with the second trimester [5]. Detection of spina bifida in the first trimester using US is difficult, as the "lemon" and "banana" signs of spina bifida appear in the second trimester [6]. However, the mid-sagittal view of the nuchal translucency (NT) scan, which is conducted in the first trimester, provides a great deal of information for uncovering malformations in the posterior fossa of the fetal head and spine [7]. One indicator of spina bifida, detected early by US, is an increased diameter of the brainstem (BS) and decreased diameter of the brainstem-occipital bone (BSOB), such that the BS to BSOB ratio exceeds the normal value $(<0.9)$, i.e., the 95 th percentile. This finding supports the use of US as a reliable screening tool for detecting open spina bifida (OSB) $[8,9]$. 
The current study used the mid-sagittal view of the fetal posterior brain in the sonogram to measure the BS, the BSOB, and the BS to BSOB ratio at the gestational age of $10^{+6}$ to $13^{+6}$ weeks, in order to establish a reference parameter for fetal tests and consistent guidelines for diagnosis of spina bifida in the first trimester. We believe that this guideline will also be useful for determining gestational age.

\section{Material and methods}

The subjects of the study were pregnant women aged between 17 and 46 years, who received prenatal care in an OB/GYN hospital ward in the Republic of Korea between April 2013 and February 2016. Signed informed consent was obtained from every subject and the study was approved by the local Ethics Committee. The gestational age (GA) was determined by the last menstrual period (LMP). Among patients at the gestational age of $10^{+6}$ to $13^{+6}$ weeks (i.e., 2,736 singleton pregnant women with a CRL between $38.9 \mathrm{~mm}$ and $78.1 \mathrm{~mm}$ ), the results of 2,621 patients were analyzed in a retrospective crosssectional study, excluding 9 patients with spontaneous abortions, 33 whose fetuses exhibited other malformations, 2 with fetuses with spina bifida, and 71 who could

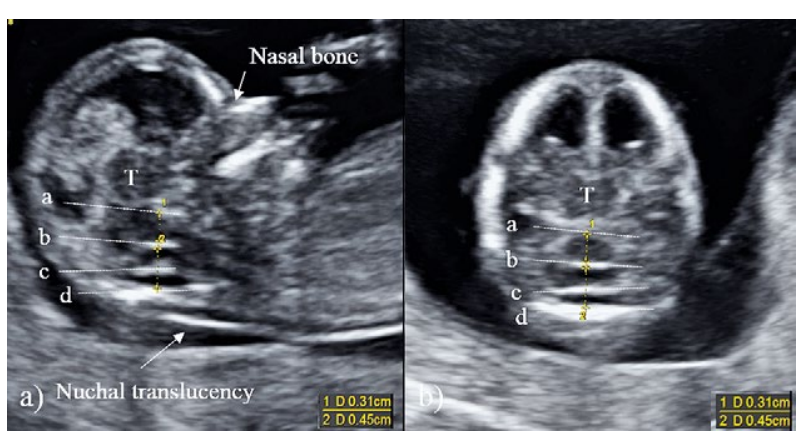

Fig 1. The fetal posterior brain showing the thalamus (T), brain stem (a-b), fourth ventricle (b-c), cisterna magna (c-d) and occipital bone (d). a) Mid-sagittal viewof the posterior brain obtained for the nuchal translucency (NT) test; b) Transverse plane of the fetal posterior brain. not be examined because of fetal position, oligohydramnios, or maternal obesity.

\section{Measurement}

A GE Voluson E8 Expert, USA system witha convex array probe frequency of $2 \sim 8 \mathrm{MHz}$ was used. A sonographer with 17 years of experience conducted all of the tests. Four lines were confirmed in the mid-sagittal view of the posterior brain and obtained for the nuchal translucency (NT) test [10].

On the fetal posterior, brain images that included the thalamus, lines a, b, c, and d indicated the posterior border of the sphenoid bone, anterior border of the fourth ventricle, posterior border of the fourth ventricle, and anterior border of the occipital bone, respectively (fig 1) [8].

When the mid-sagittal view was not attainable due to the fetal position, the four lines were confirmed and measured in the transverse plane. The longest vertical depth diameter of the $\mathrm{BS}$ and the vertical depth diameter of the BSOB were measured, and the BS to BSOB ratio calculated. The resulting values were compared with the results of the diagnostic evaluation of spina bifida.

\section{Statistical analysis}

For statistical analysis, SPSS statistics software version 22.0 (IBM, USA) was used to conduct a regression analysis of both BS and BSOB according to gestational age, to analyze correlations among variables, and to derive the predictive equation. A frequency analysis was conducted to present the percentiles of BS and BSOB values by gestational age. $P$ value $<0.05$ was considered to be significant.

\section{Results}

The characteristics of the study group are detailed in table I. The coefficient of determination $\left(\mathrm{R}^{2}\right)$ for the CRL by GA was 0.9744 . The results showed that CRL increased in proportion to GA. In contrast, the BS to BSOB ratio, which was $<1.0$ in a normal fetus, did not correlate with the CRL (Table I).

The BS diameters ranged from $1.0 \pm 0.24$ to $4.7 \pm 0.46$ $\mathrm{mm}$, and the BSOB diameters ranged from $2.9 \pm 0.36$

Table I. Characteristics of the study patients $(n=2,621)$

\begin{tabular}{llllll}
\hline & Min & Mean & Max & SD & 95\% CI \\
\hline Age (years) & 18.0 & 32.5 & 46.0 & 4.41 & $32.35-32.69$ \\
Gestational age (weeks) & 10.6 & 12.2 & 13.6 & 0.61 & $12.20-12.24$ \\
Crown rump length (mm) & 38.5 & 58.2 & 79.4 & 8.17 & $57.87-58.49$ \\
NT (mm) & 0.7 & 1.5 & 5.1 & 0.39 & $1.45-1.48$ \\
BS (mm) & 1.0 & 2.9 & 4.7 & 0.55 & $2.91-2.95$ \\
BSOB (mm) & 2.9 & 5.1 & 8.5 & 0.87 & $5.09-5.14$ \\
BS to BSOB ratio & 0.05 & 0.57 & 0.85 & 0.07 & $0.57-0.58$ \\
\hline
\end{tabular}

NT - neural tube, BS - brainstem, BSOB -brainstem-occipital bone, SD - Standard deviation 
to $8.5 \pm 0.92 \mathrm{~mm}$. As gestational age increased, the values also increased. The regression equation for BS and BSOB diameters by gestational age was obtained using the following formulas $\mathrm{BS}=0.6344 \times \mathrm{GA}-0.4289$ $\left(\mathrm{R}^{2}=0.49, \quad \mathrm{R}=0.70\right) \quad$ and $\quad \mathrm{BSOB}=1.1515 \times \mathrm{GA}-8.9672$ $\left(\mathrm{R}^{2}=0.65, \mathrm{R}=0.81\right)$. The reference range (mean, 5 th and

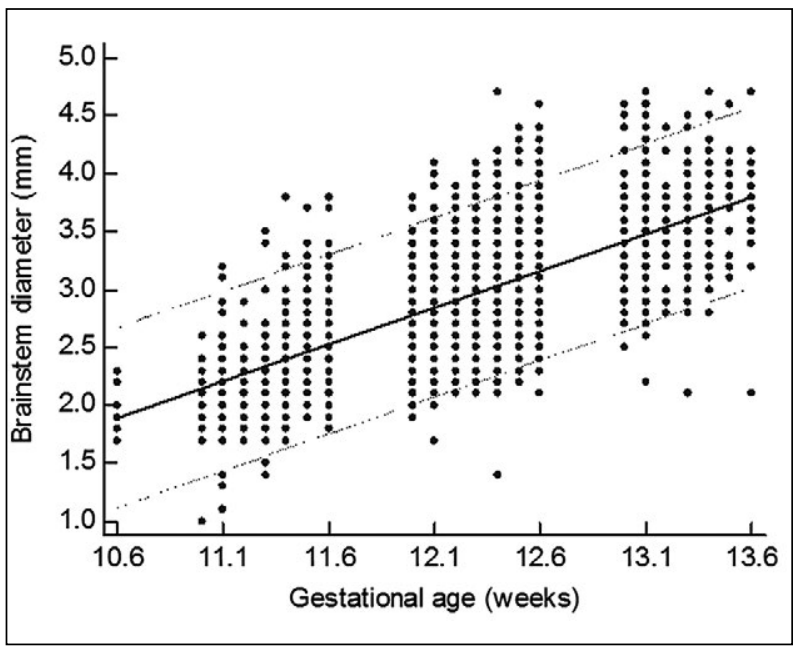

Fig 2. Scatter plot of brainstem (BS) diameter by gestational age (GA).

Table II. Percentile values of brainstem diameter by gestational age.

\begin{tabular}{llllll}
\hline GA (weeks) & \multicolumn{3}{l}{ Percentile $(\mathbf{m m})$} & & \\
\cline { 2 - 6 } & $\mathbf{5}^{\text {th }}$ & $\mathbf{2 5}^{\text {th }}$ & $\mathbf{5 0}^{\text {th }}$ & $\mathbf{7 5}^{\text {th }}$ & $\mathbf{9 5}^{\text {th }}$ \\
\hline $10+6$ & 1.7 & 1.7 & 1.9 & 2.2 & 2.3 \\
$11+0$ & 1.4 & 1.9 & 2.0 & 2.3 & 2.6 \\
$11+1$ & 1.7 & 2.0 & 2.1 & 2.3 & 2.9 \\
$11+2$ & 1.8 & 2.0 & 2.2 & 2.4 & 2.8 \\
$11+3$ & 1.7 & 2.0 & 2.3 & 2.5 & 2.9 \\
$11+4$ & 2.0 & 2.3 & 2.5 & 2.7 & 3.0 \\
$11+5$ & 2.1 & 2.3 & 2.5 & 2.6 & 3.1 \\
$11+6$ & 2.0 & 2.4 & 2.5 & 2.8 & 3.2 \\
$12+0$ & 2.1 & 2.4 & 2.6 & 2.9 & 3.2 \\
$12+1$ & 2.3 & 2.6 & 2.8 & 3.0 & 3.5 \\
$12+2$ & 2.4 & 2.6 & 2.9 & 3.2 & 3.6 \\
$12+3$ & 2.5 & 2.7 & 3.0 & 3.2 & 3.8 \\
$12+4$ & 2.4 & 2.8 & 3.0 & 3.3 & 3.9 \\
$12+5$ & 2.5 & 2.9 & 3.1 & 3.4 & 3.9 \\
$12+6$ & 2.5 & 3.0 & 3.2 & 3.5 & 4.1 \\
$13+0$ & 2.8 & 3.1 & 3.4 & 3.6 & 4.1 \\
$13+1$ & 2.8 & 3.2 & 3.4 & 3.7 & 4.4 \\
$13+2$ & 2.9 & 3.2 & 3.5 & 3.8 & 4.2 \\
$13+3$ & 2.9 & 3.3 & 3.5 & 3.8 & 4.2 \\
$13+4$ & 3.1 & 3.3 & 3.7 & 3.9 & 4.4 \\
$13+5$ & 3.1 & 3.3 & 3.8 & 4.1 & 4.3 \\
$13+6$ & 3.0 & 3.5 & 3.8 & 4.0 & 4.3 \\
\hline & & & & &
\end{tabular}

95th percentiles) are shown in fig 2 and fig 3 and the 5th, 25th, 50th, 75th and 95th percentiles by GA are presented in Table II and Table III. It is clear that the GA, BS and BSOB diameter had strong, statistically significant positive correlations $(\mathrm{p}<0.0001)$.

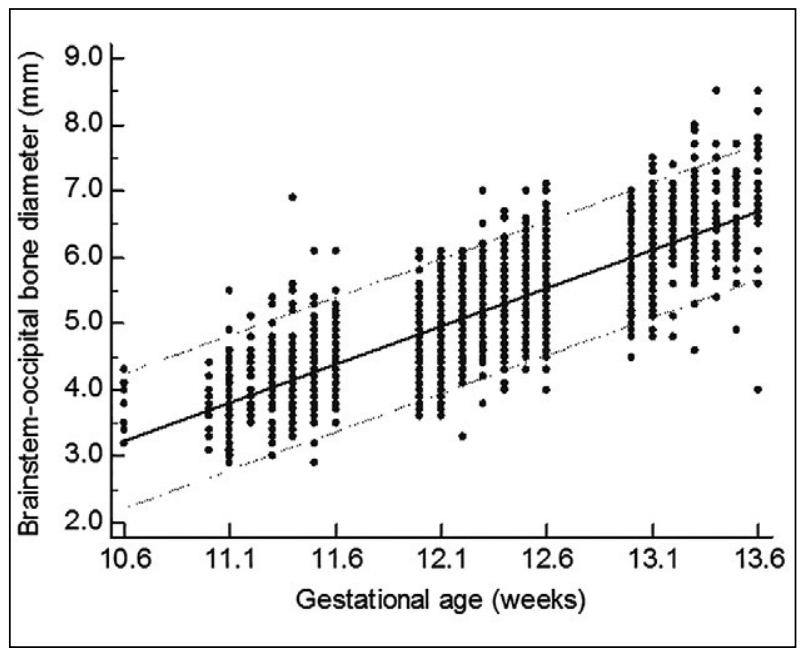

Fig 3. Scatter plot of brainstem-occipital bone (BSOB) diameter by gestational age (GA).

Table III. Percentile values of brainstem-occipital bone diameter by gestational age.

\begin{tabular}{llllll}
\hline GA (weeks) & \multicolumn{5}{l}{ Percentile (mm) } \\
\cline { 2 - 6 } & 5th & 25th & 50th & 75th & 95th \\
\hline $10+6$ & 3.2 & 3.5 & 3.7 & 4.0 & 4.3 \\
$11+0$ & 3.2 & 3.6 & 3.8 & 4.0 & 4.4 \\
$11+1$ & 3.1 & 3.5 & 3.8 & 4.0 & 4.4 \\
$11+2$ & 3.5 & 3.8 & 4.0 & 4.3 & 4.7 \\
$11+3$ & 3.3 & 3.7 & 4.1 & 4.5 & 4.9 \\
$11+4$ & 3.5 & 4.0 & 4.3 & 4.5 & 4.9 \\
$11+5$ & 3.7 & 4.0 & 4.3 & 4.6 & 5.1 \\
$11+6$ & 3.8 & 4.2 & 4.5 & 4.8 & 5.3 \\
$12+0$ & 3.8 & 4.3 & 4.6 & 4.9 & 5.4 \\
$12+1$ & 4.1 & 4.5 & 4.8 & 5.1 & 5.6 \\
$12+2$ & 4.3 & 4.7 & 5.0 & 5.4 & 5.7 \\
$12+3$ & 4.4 & 4.8 & 5.1 & 5.4 & 6.0 \\
$12+4$ & 4.5 & 5.0 & 5.3 & 5.6 & 6.1 \\
$12+5$ & 4.7 & 5.1 & 5.4 & 5.8 & 6.3 \\
$12+6$ & 4.7 & 5.3 & 5.7 & 6.0 & 6.5 \\
$13+0$ & 4.9 & 5.5 & 5.9 & 6.4 & 6.8 \\
$13+1$ & 5.1 & 5.6 & 6.0 & 6.5 & 7.0 \\
$13+2$ & 5.3 & 6.1 & 6.5 & 6.8 & 7.1 \\
$13+3$ & 5.6 & 5.9 & 6.5 & 6.9 & 7.7 \\
$13+4$ & 5.7 & 6.1 & 6.6 & 7.0 & 7.7 \\
$13+5$ & 5.4 & 6.1 & 6.5 & 7.0 & 7.7 \\
$13+6$ & 5.3 & 6.6 & 7.1 & 7.5 & 8.3 \\
\hline
\end{tabular}




\section{Discussions}

As efforts to diagnose nuchal tube malformation during the early stage of pregnancy increase, US in the fetal brain as a screening test is becoming more widely used. In 2009, Chaoui et al reported that fetuses aged 11 to 13 weeks with no visible intracranial translucency (IT), were diagnosed with spina bifida in the second trimester [11]. Afterwards, the mid-sagittal view of the NT test was used to investigate the IT value depending on the CRL $[12,13]$. In 2011, Lachmann et al reported that the BS diameter was greater than the BSOB diameter in open spina bifida in 30 fetuses who were compared with 1,000 normal fetuses in London and Berlin, and it was thus found that the BS to BSOB ratio actually increased in spina bifida [8]. Meanwhile, a study conducted in 2012 by Fatima et al reported that, in the case of aneuploidies such as trisomy 18 , trisomy 13 and triploidy, the BS to $\mathrm{BSOB}$ ratio tends to decrease due to the increase in the BSOB rather than the BS diameter [14]. In 2014, ArtuncUlkumen et al presented the normal range of IT by studying the relationship between IT, the maternal serum, and the ductus venosus pulsatility index [15]. In this study, one of the two cases diagnosed with spina bifida was putatively diagnosed with meningomyelocele as a result of the US conducted during the first trimester, but the fetus was aborted. In the other, the BS diameter was $4.0 \mathrm{~mm}$, the BSOB diameter was $3.4 \mathrm{~mm}$, and the BS to BSOB ratio was 1.18 ; i.e., $>1.0$ at $12^{+1}$ weeks of gestation. In the same case, the maternal serum AFP test, conducted at $17^{+1}$ weeks of gestation, showed a positive result, i.e. 3.351 MoM (cut off value: $2.5 \mathrm{MoM}$ ) [16], and the target US conducted during the second trimester at $18^{+1}$ weeks confirmed the "lemon" and "banana" signs [17].

The current study attempted to establish the standard value of the BS and BSOB diameter for early screening of spina bifida in Korean fetuses. Our findings support the need for routine testing during the early stage of pregnancy. For this study, the mean, standard deviation, and $95 \%$ confidence interval of the BS and BSOB diameters at $10^{+6}$ to $13^{+6}$ weeks of gestation were obtained. The results of the study showed that in 2,621 normal fetuses, the CRL increased in proportion to the $\mathrm{GA}\left(\mathrm{R}^{2}=0.9744\right)$. The BS and BSOB diameters also increased in proportion to the GA. The BS diameters ranged from 1.0 0.24 to $4.7 \pm 0.46 \mathrm{~mm}$, and the BSOB diameters ranged from $2.9 \pm 0.36$ to $8.5 \pm 0.92 \mathrm{~mm}$. As gestational age increased, BS and BSOB increased with statistically significant differences $(\mathrm{p}<0.0001)$. In normal patients, the BS diameter was smaller than the BSOB diameter, while in the spina bifida case, the BS to BSOB ratio was greater than 1.0. The BS and BSOB diameters established in this study, along with routine NT tests conducted in the first trimester, are expected to be useful as screening criteria for evaluating the posterior brain for spina bifida.

One limitation of this study was that $2.6 \%$ of patients could not be studied because of the fetal position, oligohydramnios, or maternal obesity. Some subjective error is also highly likely because only one sonographer conducted all of the tests. However, if the scanning method is changed, or if the test is conducted while watching the movement of the fetus over a sufficient period of time, more precise measurements can be realized.

\section{Conclusions}

In conclusion, evaluation of the posterior brains of the fetuses of Korean pregnant women, using the reference values for the BS and BSOB diameters measured using the mid-sagittal view at a GA between $10^{+6}$ to $13^{+6}$ weeks can be a useful tool for detection of spina bifida.

\section{Conflict of interest: none}

\section{References}

1. Sebire NJ, Noble PL, Thorpe-Beeston JG, Snijders RJ, Nicolaides KH. Presence of the 'lemon' sign in fetuses with spina bifida at the 10-14 week scan. Ultrasound Obstet Gynecol 1997;10:403-405.

2. Tortori-Donati P, Rossi A, Cama A. Spinal dysraphism: a review of neuroradiological features with embryological correlations and proposal for a new classification. Neuroradiology 2000;42:471-491.

3. Bradley LA, Palomaki GE, McDowell GA. Technical standards and guidelines: prenatal screening for open neural tube defects. Genet Med 2005;7:355-369.

4. Oh BH, Lee JM, Lee KH, Kwon MS. Determination of amniotic fluid alpha-fetoprotein and acetylcholinesterase for prenatal diagnosis of open neural tube defects. Korean J Obstet Gynecol 1999;42:759-764.

5. Bredaki FE, Poon LC, Birdir C, Escalante D, Nicolaides $\mathrm{KH}$. First-trimester screening for neural tube defects using alpha-fetoprotein. Fetal Diagn Ther 2012;31:109-114.

6. Liu F, Zhang Z, Lin X, et al. Development of the human fetal cerebellum in the second trimester: a post mortem magnetic resonance imaging evaluation. J Anat 2011;219:582588.

7. Egle D, Strobl I, Weiskopf-Schwendinger V, et al. Appearance of the fetal posterior fossa at $11+3$ to $13+6$ gestational weeks on transabdominal ultrasound examination. Ultrasound Obstet Gynecol 2011;38:620-624.

8. Lachmann R, Chaoui R, Moratalla J, Picciarelli G, Nicolaides KH. Posterior brain in fetuses with open spina bifida at 11 to 13 weeks. Prenat Diagn 2011;31:103-106.

9. Chaoui R, Nicolaides KH. Detecting open spina bifida at the 11-13 week scan by assessing intracranial translucency 
and the posterior brain region: mid-sagittal or axial plane? Ultrasound Obstet Gynecol 2011;38:609-612.

10. Iuculano A, Zoppi MA, Piras A, Arras M, Monni G. Brain stem/brain stem occipital bone ratio and the four-line view in nuchal translucency images of fetuses with open spina bifida. J Matern Fetal Neonatal Med 2014;10:1-4.

11. Chaoui R, Benoit B, Mitkowska-Wozniak H, Heling KS, Nicolaides KH. Assessment of intracranial translucency (IT) in the detection of spina bifida at the 11-13 week scan. Ultrasound Obstet Gynecol 2009;34:249-252.

12. Liu M, Liu Y, Li ZH, Yu D. Screening for fetal spina bifida aperta by the ultrasound and intracranial translucency examinations at 11-13(+6) weeks of gestation. Cell Biochem Biophys 2015;72:439.

13. Yuksel MA, Arisoy R, Erdogdu E, Imamoglu M, Yayla M, Sen C. Relationship between first trimester visualization of the intracranial translucency and spina bifida. Arch Gynecol Obstet 2015;291:513-516.

14. Ferreira AF, Syngelaki A, Smolin A, Vayna AM, Nicolaides $\mathrm{KH}$. Posterior brain in fetuses with trisomy 18, trisomy 13 and triploidy at 11 to 13 week's gestation. Prenat Diagn 2012;32:854858.

15. Artunc-Ulkumen B, Pala HG, Uyar Y, Bulbul-Baytur Y, Koyuncu FM 1. Normal range of intracranial translucency in healthy Turkish pregnancies and its association with first trimester maternal serum biochemistry and ductus venosus pulsatility index. Ginekol Pol 2014;85:738-741.

16. Estimating an individual's risk of having a fetus with open spina bifida and the value of repeat alpha-fetoprotein testing. J Epidemiol Community Health 1982;36:87-95.

17. Benacerraf BR, Stryker J, Frigoletto FD Jr. Abnormal US appearance of the cerebellum (banana sign): indirect sign of spina bifida. Radiology 1989;171:151-153. 